\title{
ZNF277 regulates ovarian cancer cell proliferation and invasion through inhibition of PTEN
}

This article was published in the following Dove Medical Press journal: OncoTargets and Therapy

\author{
Zhengling Liu \\ Zonglan Xu \\ Yonghui Tian \\ Hua Yan \\ Yanyan Lou
}

Department of Gynaecology, Linyi Central Hospital, Linyi, Shandong,

276400, People's Republic of China
Correspondence: Yonghui Tian Department of Gynaecology, Linyi Central Hospital, 17th Jiankang Road, Yishui, Linyi, Shandong, 276400, People's Republic of China Email tianyonghuipo@I63.com

\begin{abstract}
Background: ZNF277 is a transcription factor that is overexpressed in several cancers. However, its clinical role in ovarian cancer (OC) has not been reported yet. The present study aims to investigate the expression of ZNF277 in patients with OC, and to reveal the effects of ZNF277 on the proliferation, migration, and invasion of OC cells.

Methods: Using The Cancer Genome Atlas database, we found that higher expression of ZNF277 was correlated with poorer survival times of OC patients. This study used functional experiments, such as Cell Counting Kit-8 assay, colony formation assay, wound healing assay, and transwell invasion assay. Mechanistically, using quantitative chromatin immunoprecipitation assay, luciferase reporter assay, quantitative reverse-transcription PCR, and Western blot we identified the potential mechanism.

Results: We confirmed for the first time that the expression of ZNF277 is significantly increased in OC tissues and cell lines and that it is closely associated with the adverse clinical features of OC patients. We demonstrated that overexpression of ZNF277 potentiated the proliferation, migration, and invasion of SKOV3 and OVCAR3 loss-of-function experiments showed that the silencing of ZNF277 reduced the proliferation, migration, and invasion of OC cells. Mechanistically, using quantitative chromatin immunoprecipitation assay, luciferase reporter assay, quantitative reverse-transcription PCR, and Western blot we identified that PTEN was a direct downstream target for ZNF277. PTEN expression antagonized the tumor-promoting function of ZNF277.

Conclusion: Taken together, the results of the current study demonstrated that ZNF277 exerted a promoting role in the progression of $\mathrm{OC}$ and might act as a promising biomarker and therapeutic target for OC patients.
\end{abstract}

Keywords: ZNF277, ovarian cancer, proliferation, migration, invasion, PTEN

\section{Introduction}

Ovarian cancer (OC) is a common malignant tumor with a serious threat to the health of females all over the world. ${ }^{1,2}$ Despite numerous advances in integrated therapeutic approach, including surgery, chemotherapy, radiotherapy, and immune therapy, the overall survival rate remains unsatisfactory with a mean 5 -year survival rate of $\sim 40 \%{ }^{3,4}$ Since frequent relapse and metastasis result in poor prognosis, the examination of the underlying mechanisms involved in OC remains to be elucidated. ${ }^{5}$

PTEN, a dual lipid/protein phosphatase, ${ }^{6}$ works as a well-known tumor suppressor by negatively regulating the AKT signaling pathway, ${ }^{7}$ and has been found to play important roles in the progression of various human cancers including OC. ${ }^{8,9}$ Accumulating studies have confirmed that several miRNAs such as MiR-200a, ${ }^{10} \mathrm{MiR}-19 \mathrm{~b},{ }^{11}$ and MiR-216a ${ }^{12}$ could promote the invasion and migration of OC by targeting the $3^{\prime}$ UTR of PTEN, thus suppressing the PTEN/AKT pathway. However, the suppression of PTEN by the binding of transcription factors to the promoter region in $\mathrm{OC}$ remains unclear. 
ZNF277 contains five repeats of a $\mathrm{C}_{2} \mathrm{H}_{2}$-type zinc finger motif, with RNA polymerase II transcription factor activity, sequence-specific DNA binding activity, and is localized to human chromosome 7q31.1.13 With evolutionary conservation, ZNF277 has been reported to be a critical regulator in differentiation and plays an important role in cellular senescence and cancer. ${ }^{14,15}$ Till now, the function of ZNF277 in OC remains to be investigated. The objective of the study is to offer insights into ZNF277 function and suggest ZNF277 as a potential target for $\mathrm{OC}$.

\section{Materials and methods}

\section{Tissue samples and cell lines}

All tissue samples were obtained from the Department of Gynaecology, Linyi Central Hospital (Shandong, China) between February 2011 and December 2013. Eighty-six tissue specimens and their corresponding adjacent non-tumor tissues were obtained from female patients, with age ranging from 40 to 55 years, who underwent tumor surgical resections. The tissues were stored at $-80^{\circ} \mathrm{C}$ for later use. All patients had not undergone chemo- or radiotherapy before surgery. The study was approved by the Clinical Research Ethics Committee of Linyi Central Hospital (No 2013-111). The informed consent and written approvals were obtained from all the participants.

The human OC cell lines SKOV3, OVCAR3, A2780, and CAOV3 were purchased from the American Type Culture Collection (ATCC, Manassas, VA, USA). Normal human ovarian surface epithelial cells (HOSEPiCs) were purchased from the Cell Bank of Type Culture Collection of Chinese Academy of Sciences (Shanghai, China). The relative cells were cultured in DMEM or RPMI-1640, respectively (Thermo Fisher Scientific, Waltham, MA, USA), containing 10\% FBS, $100 \mathrm{U} / \mathrm{mL}$ penicillin, and $100 \mathrm{mg} / \mathrm{mL}$ streptomycin (Thermo Fisher Scientific), and were cultured at $37^{\circ} \mathrm{C}$ in a humidified atmosphere with $5 \% \mathrm{CO}_{2}$.

\section{Cell transfection}

Lipofectamine $^{\circledR} 2000$ was obtained from Thermo Fisher Scientific. Transfection was performed according to the protocol recommended by the manufacturer. Forty-eight hours after transfection, the cells were collected and used for further experiments. The sequences of the ZNF277 short hairpin RNA (shZNF277) were purchased from GenePharma Co., Ltd. (Shanghai, China).

\section{RNA extraction and quantitative reverse- transcription PCR (RT-qPCR)}

Total RNA was extracted using $1 \mathrm{~mL}$ of TRIzol $^{\circledR}$ reagent (Thermo Fisher Scientific) from $100 \mu \mathrm{g}$ of human OC tissues for each specimen or OC cell lines according to the manu- facturer's protocol. UV spectrophotometer (Thermo Fisher Scientific) was used to monitor the total RNA concentration and purity. A PrimeScript RT reagent kit (Thermo Fisher Scientific) was used to reverse-transcribe RNA into cDNAs. RT-qPCR system (ABI 7500; Applied Biosystems, Foster City, CA, USA) with SYBR Green Master Mix (Toyobo, Tokyo, Japan) was used for qPCR. GAPDH was used as a reference gene. The thermocycler conditions were as follows: $95^{\circ} \mathrm{C}$ for 45 seconds and then 40 cycles at $90^{\circ} \mathrm{C}$ for 15 seconds, $60^{\circ} \mathrm{C}$ for 10 seconds, and $72^{\circ} \mathrm{C}$ for 45 seconds. The primers used were as follows: ZNF277: 5'-GAACATGCTTTCAACA TTGG-3' (forward) and 5'-ATCAGACCAGTCATCT TCCT-3' (reverse); GAPDH: 5'-AGCCACATCGCTCAG ACAC-3' (forward) and 5'-GCCCAATACGACCAAATCC-3' (reverse). The $2-\Delta \Delta C$ T method was performed to demonstrate the relative expression of all the target genes. ${ }^{16}$

\section{Western blot}

Equal amount of protein $(30 \mu \mathrm{g})$ in each group was separated by $10 \%$ SDS-PAGE and then transferred to nitrocellulose membranes. Next, the membranes were blocked with $5 \%$ non-fat milk in Tris-buffered saline with $0.1 \%$ Tween (Thermo Fisher Scientific) for 1 hour at room temperature. The membrane was incubated with primary antibodies at $37^{\circ} \mathrm{C}$ for 2 hours, and then incubated with horseradish peroxidaseconjugated anti-rabbit secondary antibodies (Sigma-Aldrich Co., St Louis, MO, USA) at $37^{\circ} \mathrm{C}$ (dilution, 1:5,000) for 2 hours. The protein bands were detected using Pierce Electrochemiluminescence Plus Substrate (Thermo Fisher Scientific) on Kodak film developer (Fujifilm, Tokyo, Japan). The primary antibodies used were as follows: PTEN (ab32199; Abcam, Cambridge, UK) and ZNF277 (ab96299; Abcam).

\section{Luciferase reporter assay}

Luciferase reporter assay was performed using the DualLuciferase Reporter Assay System (Promega Corporation, Madison, WI, USA) according to the manufacturer's protocol. The relative cells were cultured in 24 -well plates at $37^{\circ} \mathrm{C}$ overnight, and then co-transfected with luciferase reporter plasmids with wild-type or mutant PTEN reporter constructs together with the ZNF277 overexpression or knockdown construct which were purchased from Genepharma (Shanghai, China). Forty-eight hours after transfection, cells were harvested and the luciferase activity was measured.

\section{Quantitative chromatin immunoprecipitation assay}

Briefly, cells were cross-linked with $1 \%$ formaldehyde, sonicated, and pre-cleared. After incubation with 5-10 $\mu \mathrm{g}$ 
of the appropriate antibody, protein A/G Sepharose CL-4B beads were added into the immune complex. The beads were then washed in buffers and DNA was eluted for quantitative chromatin immunoprecipitation assay.

\section{Proliferation assay}

The $100 \mu \mathrm{L}$ OV cells $\left(5 \times 10^{3}\right.$ cells/well $)$ were seeded in 96-well culture plates (Corning Incorporated, New York, NY, USA), and after culturing for 24, 48, 72, and 96 hours at $37^{\circ} \mathrm{C}, 20 \mu \mathrm{L}$ of MTT reagent (Dojindo Molecular Technologies, Inc., Kumamoto, Japan) was added and plates were incubated for another 4 hours. The supernatant was removed, and $150 \mu \mathrm{L}$ of dimethyl sulfoxide was added into each well. The absorbance was measured using an ELX-800 spectrometer reader (Omega Bio-Tek, Inc., Norcross, GA, USA) at $490 \mathrm{~nm}$. The OD values represent the relative proliferation level of the cells. The experiments were performed in triplicate.

\section{Colony formation assay}

The transfected cells were seeded into six-well plates with 1,000 cells/well, and cultured at $37^{\circ} \mathrm{C}$ with $5 \% \mathrm{CO}_{2}$ for 14 days. Then, colonies were fixed with $100 \%$ methanol for 30 minutes and stained with $0.2 \%$ crystal violet for 15 minutes (Thermo Fisher Scientific) at room temperature. The total number of colonies ( $>50$ cells) was counted using light microscopy (magnification, $\times 40$; Olympus, Tokyo, Japan). The experiment was performed in triplicate.

\section{Wound healing assay}

In order to detect the migration ability, transfected cells were trypsinized and seeded into a 12 -well plate at the density of $2 \times 10^{5}$ cells per well. After overnight incubation, we scratched the confluent monolayer across the plate using a $10 \mu \mathrm{L}$ pipette tip. The wells were washed with PBS for three times to make sure no suspending cells existed in the wound areas. The wounded monolayer was photographed immediately. Subsequently, the cells were incubated in low-serum medium for 24 hours and the wound images were taken again. The migration ability was calculated by the ratio of the healing width at 24 hours to the wound width at 0 hours.

\section{Transwell invasion assay}

Invasion assays were performed using transwell inserts ( $8 \mu \mathrm{M}$ pore size; Costar, Cambridge, MA, USA). The transwell inserts were coated with $100 \mu \mathrm{L}$ of Matrigel (BD Biosciences) and $500 \mu \mathrm{L}$ of FBS-free DMEM was added into 24-well plates. The OC cells were added into the top chamber, while $1,000 \mu \mathrm{L}$ of DMEM with $20 \% \mathrm{FBS}$ was added into the bottom chamber. After incubation for 48 hours, $4 \%$ paraformaldehyde was used to fix the invasive cells for 20 minutes, and then $0.5 \%$ crystal violet (Beyotime) was used for staining. The cells were counted under an inverted microscope (magnification, $\times 40$, TS100; Nikon, Tokyo, Japan) in five random fields of view. The experiment was repeated three times.

\section{Statistical analysis}

All statistical analyses were performed using SPSS 17.0 software (SPSS, Inc., Chicago, IL, USA). Data were presented as the mean $\pm \mathrm{SD}$. The log-rank test was used for the Kaplan-Meier curve to evaluate the association between the expression of ZNF277 and the survival rate. Parametric statistics were carried out, Student's $t$-test was used to compare differences between the two groups, and two-way ANOVA was used for comparison amongst three groups or between two groups with two factors, followed by a least significant difference post hoc comparison test. $P<0.05$ was considered to indicate a statistically significant difference. All experiments were repeated at least three times.

\section{Results \\ ZNF277 expression was upregulated in ovarian cancer tissues and cell lines}

As we know, the expression profile of ZNF277 in OC has not been elucidated yet. In the present study, we first use the online database of the Human Protein Atlas. The expression pattern of ZNF277 in OC tissues and normal ovary was examined by using IHC data from https://www. proteinatlas.org/, as Uhlen et al reported. ${ }^{17}$ As shown in Figure 1A, ZNF277 was upregulated in OC tissues. Further, we used the The Cancer Genome Atlas (TCGA) database to analyze the correlation between the expression of ZNF217 and the prognosis. Survival analysis showed that patients with ZNF277 overexpression had a significantly shorter overall survival (OS) time compared with those with lower ZNF277 expression (Figure 1B). RT-qPCR was used to detect the expression of ZNF277 mRNA in the primary OC tissues and tumor-adjacent normal tissues obtained from patients treated in Linyi Central Hospital. The OC tissues had significantly increased expression of ZNF277 mRNA compared with the adjacent normal tissues, and the difference was statistically significant (Figure 1C). As shown in Table 1, higher expression of ZNF277 was correlated with the lymph node metastasis, tumor size, and Federation Internationale of Gynecologie and Obstetrigue stage. Further, the expression of ZNF217 mRNA was assessed in SKOV3, 


\section{A Adjacent normal tissues}

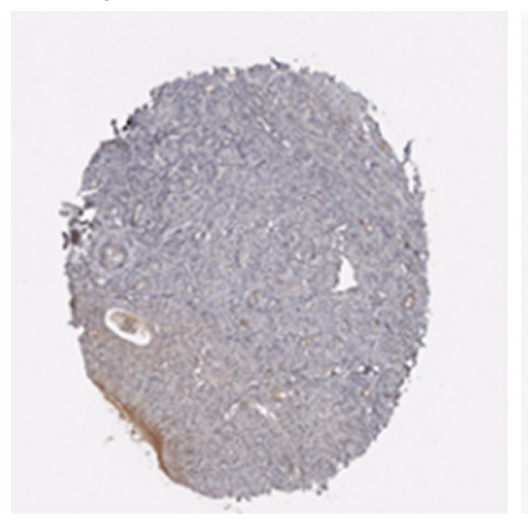

\section{Ovarian cancer tissues}

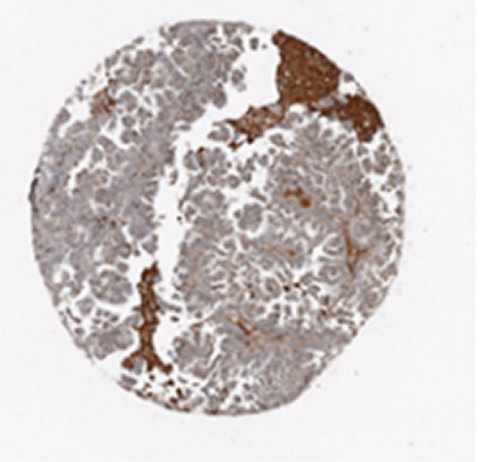

B

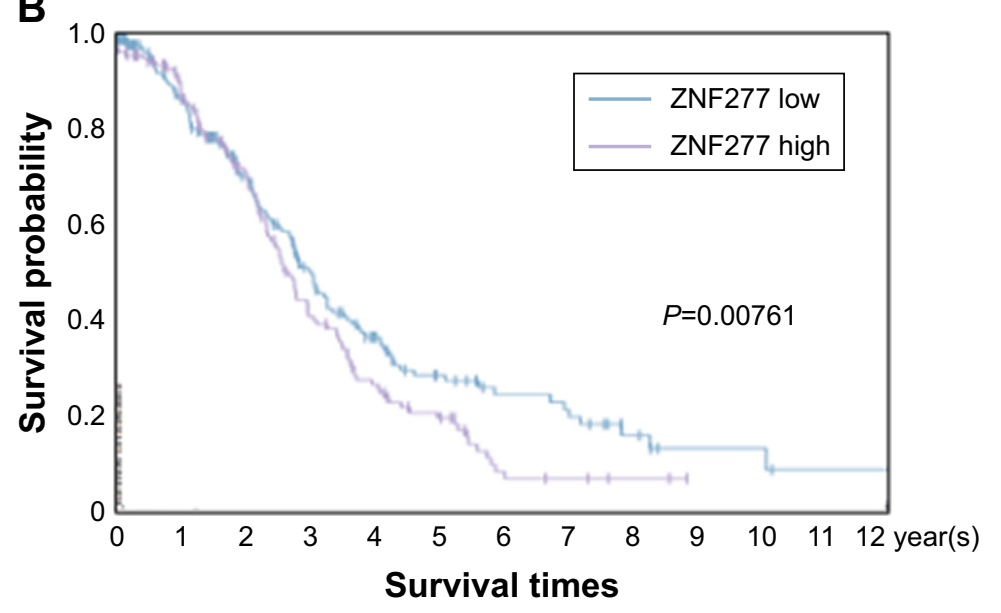

C

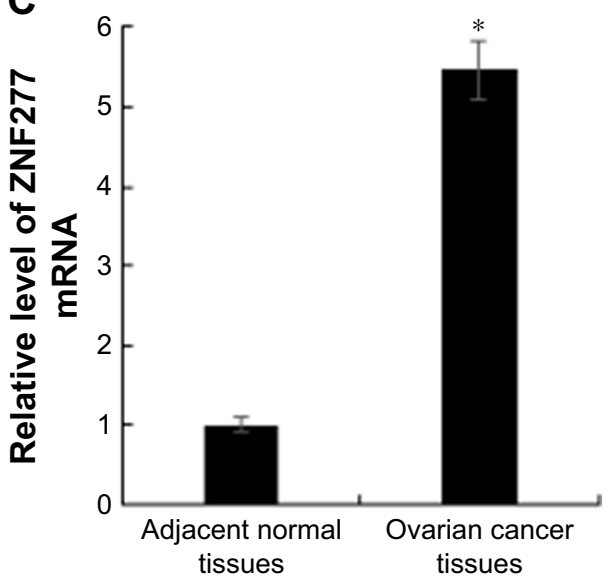

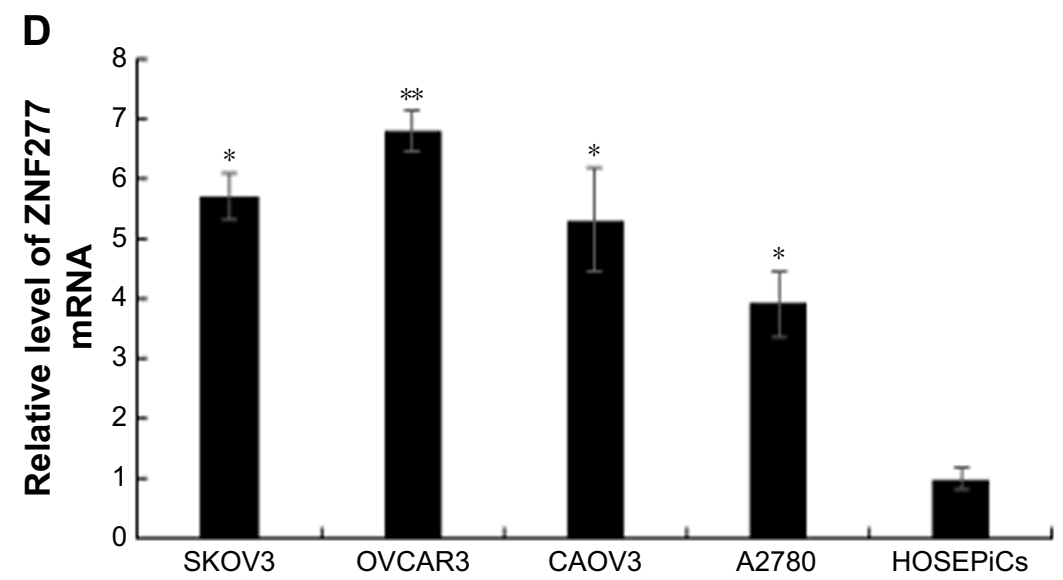

Figure I ZNF277 expression was upregulated in ovarian cancer (OC) tissues and cell lines. (A) The Human Protein Atlas database was used to detect the expression of ZNF277 in OC tissues and normal ovary tissues. (B) TCGA database was used to detect the survival times of patients with high ZNF277 expression and those with low ZNF277 expression, and Kaplan-Meier curves were drawn. (C) RT-qPCR was used to reveal the expression of ZNF277 in OC tissues and tumor-adjacent normal tissues, and the reference protein GAPDH was used as a negative control $(n=3)$. Data are presented as the mean $\pm S D$ of three independent experiments $(n=3 ; * P<0.05$ vs tumor-adjacent normal tissues). (D) RT-qPCR was used to reveal the expression of ZNF277 in the OC cell lines SKOV3, OVCAR3, CAOV3, and A2780 compared to the human ovarian surface epithelial cells (HOSEPiCs). Results are presented as the mean \pm standard error of three independent experiments $(* P<0.05$, $* * P<0.01$ vs $\mathrm{HOSEPiCs})$. Abbreviations: TCGA, The Cancer Genome Atlas; RT-qPCR, quantitative reverse-transcription PCR.

\section{OVCAR3, A2780, CAOV3, and HOSEPiCs cells. As shown} in Figure 1D, ZNF277 expression was upregulated in OC cell lines compared with HOSEPiCs. These results indicated that ZNF277 might have an important role in OC progression.

\section{ZNF277 facilitated the proliferation abilities of OC cells}

To further explore the function of ZNF277 in the proliferation and invasion of epithelium ovarian cancer, we transfected 
Table I The clinicopathologic variables identified in 86 ovarian cancer patients

\begin{tabular}{|c|c|c|c|c|}
\hline \multirow[t]{2}{*}{ Variables } & \multirow[t]{2}{*}{ No $(n=86)$} & \multicolumn{2}{|c|}{$\begin{array}{l}\text { ZNF277 protein } \\
\text { expression }\end{array}$} & \multirow[t]{2}{*}{$P$-value } \\
\hline & & $\begin{array}{l}\text { Low } \\
(n=32)\end{array}$ & $\begin{array}{l}\text { High } \\
(n=54)\end{array}$ & \\
\hline \multicolumn{4}{|l|}{ Age (years) } & \multirow[t]{3}{*}{0.158} \\
\hline$<50$ & 38 & 11 & 27 & \\
\hline$\geq 50$ & 48 & 21 & 27 & \\
\hline \multicolumn{4}{|c|}{ Ascites (mL) } & \multirow[t]{3}{*}{0.729} \\
\hline$\leq 100$ & 49 & 19 & 30 & \\
\hline$>100$ & 37 & 13 & 24 & \\
\hline \multicolumn{4}{|c|}{ Serum CA-I25 (U/mL) } & \multirow[t]{3}{*}{0.468} \\
\hline$\leq 35$ & 44 & 18 & 26 & \\
\hline$>35$ & 42 & 14 & 28 & \\
\hline \multicolumn{4}{|l|}{ Tumor size } & \multirow[t]{3}{*}{0.014} \\
\hline$\leq 3 \mathrm{~cm}$ & 39 & 20 & 19 & \\
\hline$>3 \mathrm{~cm}$ & 47 & 12 & 35 & \\
\hline \multicolumn{4}{|c|}{ Lymph node metastasis } & \multirow[t]{3}{*}{0.012} \\
\hline Negative & 44 & 22 & 22 & \\
\hline Positive & 42 & 10 & 32 & \\
\hline \multicolumn{4}{|l|}{ FIGO stage } & \multirow[t]{3}{*}{0.044} \\
\hline I-II & 39 & 19 & 20 & \\
\hline III-IV & 47 & 13 & 34 & \\
\hline
\end{tabular}

Note: The expression of ZNF277 is based on the mRNA level, and the mean expression is used as cutoff to define the high or low expression of ZNF277.

Abbreviations: CA, cancer antigen; FIGO, Federation Internationale of Gynecologie and Obstetrigue.

ZNF277 plasmid or ZNF277 siRNA into SKOV3 and OVCAR3 cells. The transfection efficiency was measured by RT-qPCR (Figure 2A and B). Cell Counting Kit-8 (CCK-8) assays were performed to assess the function of ZNF277 on OC cell proliferation. Compared with the control group, SKOV3 and OVCAR3 cells transfected with ZNF277 exhibited significantly increased proliferation ability, while knockdown of ZNF277 decreased the proliferation in a timedependent manner (Figure 2C and D). Similarly, colony formation assays revealed that the number of cell colonies generated was obviously decreased in the siZNF277 transfected groups, while in the ZNF277 overexpression groups, the colonies were significantly increased (Figure 2E and F).

\section{ZNF277 promoted migration and invasion in OC cells}

To fully explore the putative biological functions of ZNF277 using wound healing assay, the cells were treated with ZNF277 and migration pattern was studied for 24 hours. The results showed that the migration capacity of SKOV3 and OVCAR3 cells increased, while knockdown of ZNF277 decreased cell migration (Figures $3 \mathrm{~A}$ and B and S1). Transwell assays were then performed to monitor the invasive function of ZNF277 on OC cells. As shown in Figure 3C and D, in response to ZNF277 knockdown, the invasive ability of SKOV3 and OVCAR3 cells in vitro was specifically suppressed.

\section{PTEN was a direct downstream target of ZNF277 in OC cells}

To elucidate the molecular mechanisms underlying the promotion effect of ZNF277 on OC progression, we further identified the key target genes of ZNF277 in SKOV3 and OVCAR 3 cells using qChIP assay; among them, the binding of PTEN is the most remarkable one (Figure 4A). To further confirm that ZNF277 could directly target the promoter of PTEN, luciferase reporter assay was performed in SKOV3 and OVCAR3 cells. As shown in Figure 4B, forced expression of ZNF277 in the two cell lines significantly suppressed the luciferase activity of wild-type promoter of PTEN. On the other hand, inhibition of ZNF277 obviously enhanced the luciferase activity of promoter (Figure 4C), while the overexpression or knockdown of ZNF277 had no significant influence on the mutant promoter of PTEN (Figure 4D). So, we can hypothesize that ZNF277 could regulate PTEN at transcription level. The mRNA and protein expression of PTEN was significantly downregulated in response to ZNF277 overexpression (Figure 4E), while the mRNA and protein level of PTEN increased following the silencing of ZNF277 (Figure 4F).

\section{ZNF277 promoted OC cell proliferation by targeting PTEN}

After confirming PTEN was a direct target of ZNF277 in $\mathrm{OC}$, we further investigated whether PTEN has mediator effects on the function of ZNF277 in OC cells. Firstly, PTEN overexpression construct was transfected into SKOV3 and OVCAR3 cells, and RT-qPCR analysis was used to confirm the transfection efficiency of PTEN (Figure 5A). Then CCK-8 assay showed that overexpression of PTEN could reduce the proliferation ability and partly abrogate the promoting effects of ZNF277 of the two cells (Figure 5B and $\mathrm{C}$ ). Consistent with the proliferation assay, overexpression of PTEN in cells transfected with ZNF277 also resulted in retarded colony formation (Figure 5D and $\mathrm{E}$ ).

\section{ZNF277 regulated OC cell migration and invasion through PTEN}

Previous reports have indicated that PTEN has an important role in cell migration and invasion. Therefore, we probed 
A

SKOV3

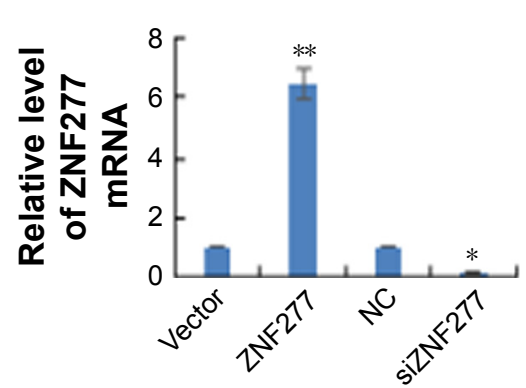

C

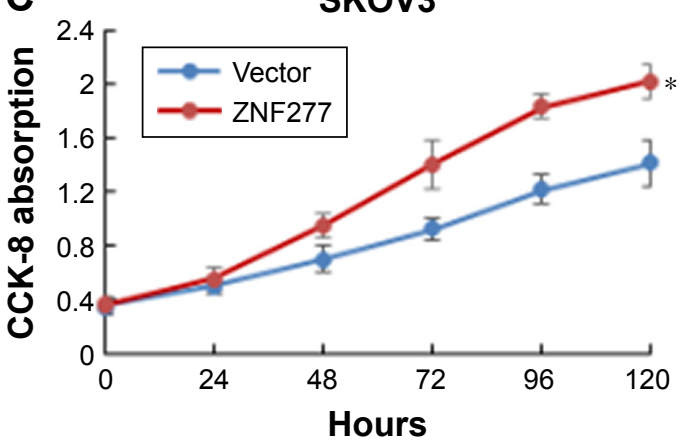

D

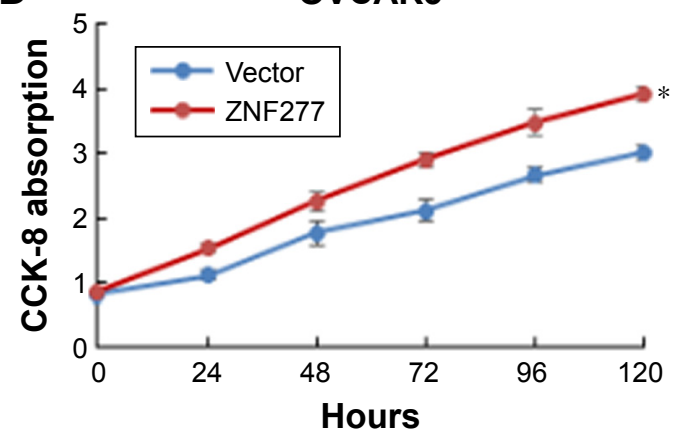

B OVCAR3
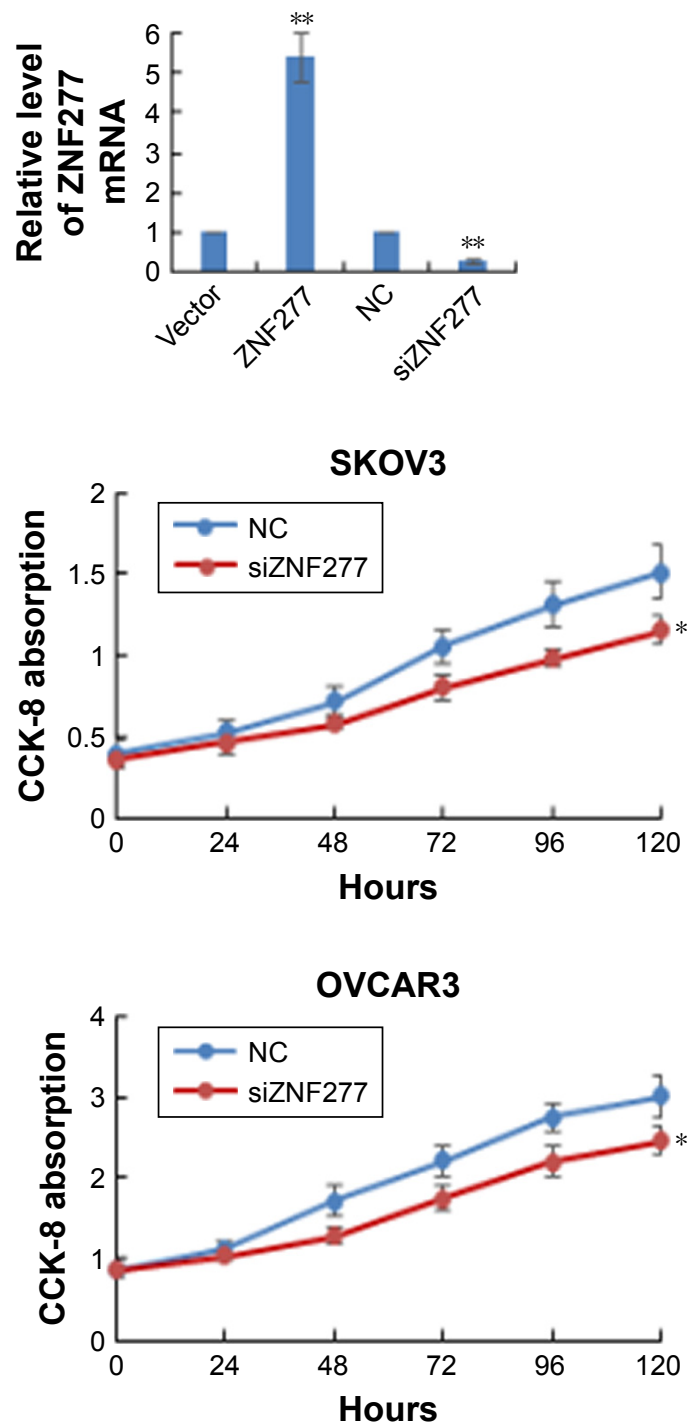

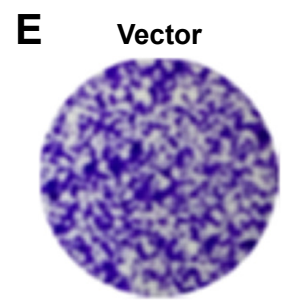

NC

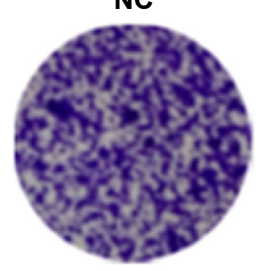

ZNF277

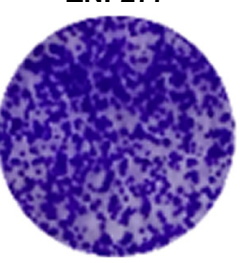

siZNF277

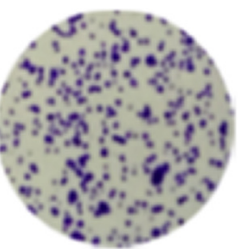

$\mathbf{F}$

SKOV3

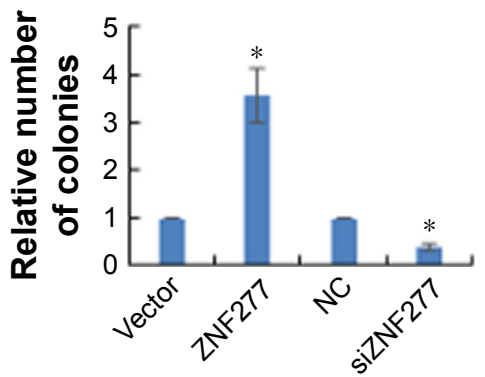

OVCAR3

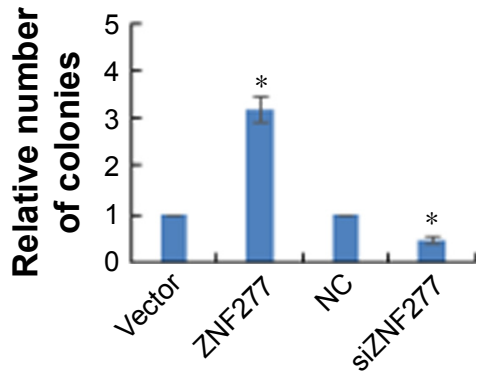

Figure 2 ZNF277 facilitated the proliferation ability of OC cells. (A) RT-qPCR was used to analyze the mRNA expression levels of ZNF277 after exposure to the ZNF277 plasmid or ZNF277-specific siRNA in SKOV3 and OVCAR3 cells (B). (C) ZNF277 affected the growth rate of OC cells. Cell growth viability was assayed in SKOV3 and OVCAR3 cells (D) transfected with vector, ZNF277 plasmid, NC, and siZNF277 using CCK-8 at 0, 24, 48, 72, and 96 hours time points. (E) ZNF277 facilitated the colony formation of OC cells. Colony formation was performed in stably overexpressing ZNF277 and in ZNF277 knockdown SKOV3 and OVCAR3 cells (F). Results are presented as mean $\pm S D$, and error bars represent the mean $\pm S D$ of three independent experiments. $* P<0.05, * * P<0.01$ as compared with the control group.

Abbreviations: OC, ovarian cancer; RT-qPCR, quantitative reverse-transcription PCR; NC, negative control; CCK-8, Cell Counting Kit-8. 
A
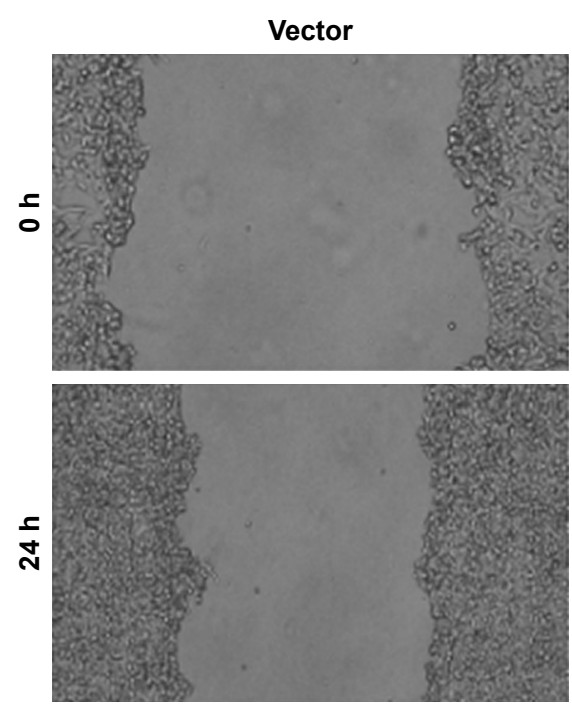

B

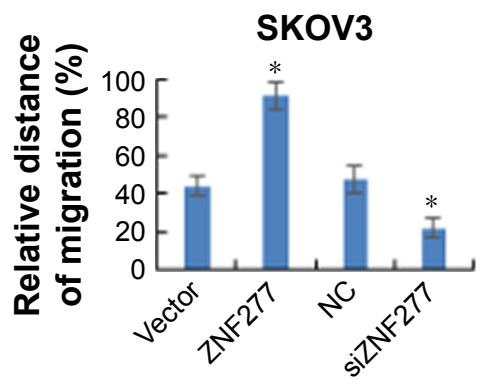

SKOV3
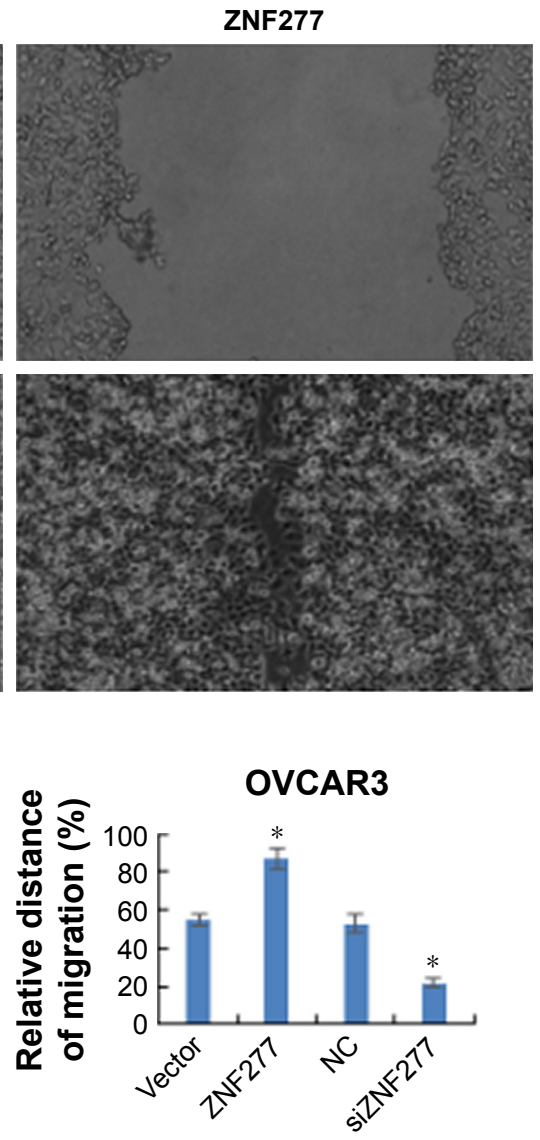

C
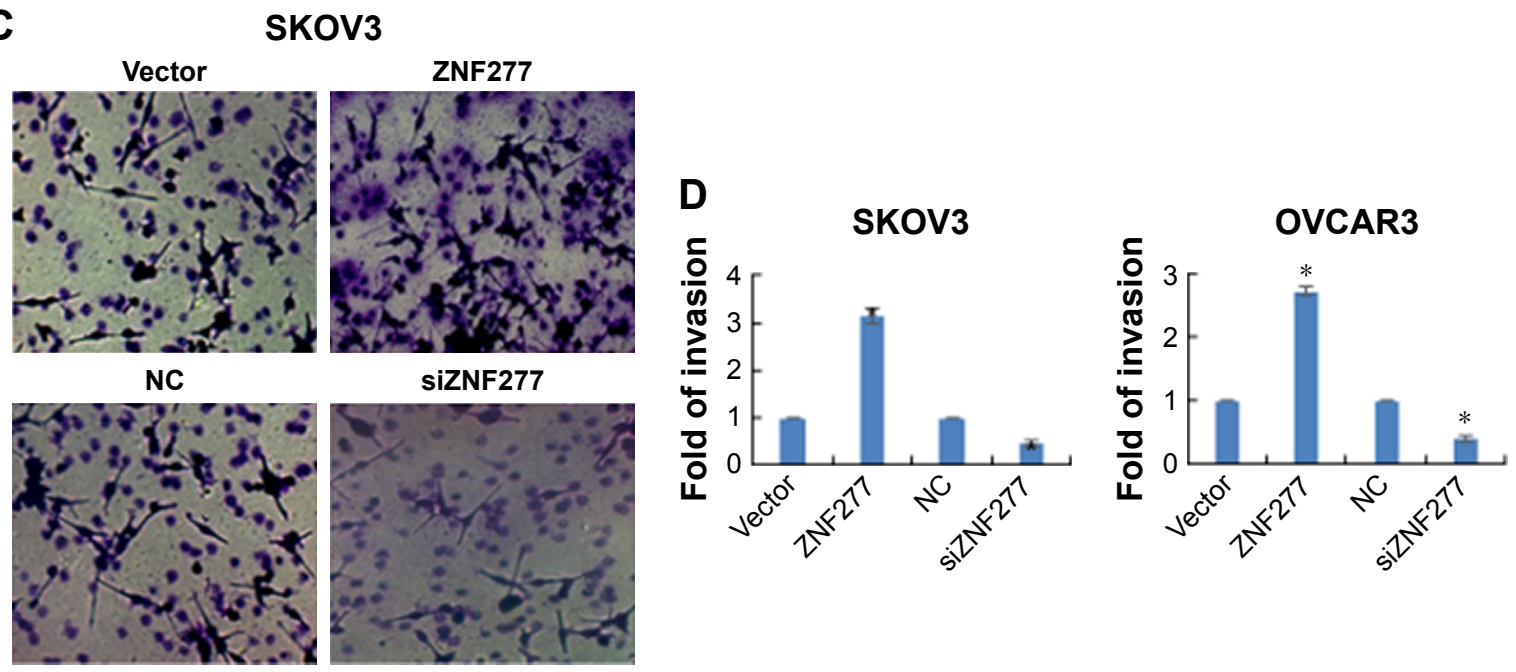

Figure 3 ZNF277 promoted migration and invasion in OC cells. (A) ZNF277 promoted migration in OC cells. Wound healing assay was performed in SKOV3 and OVCAR3 cells (B) following transfection with vector, ZNF277 plasmid, NC, and siZNF277. Analysis of the wound closure rate is shown, and data are presented as the mean \pm SD of at least three independent experiments. (C) ZNF277 promoted invasion in OC cells. Transwell assays were used to measure the effect of ZNF277 on cell invasion in SKOV3 and OVCAR3 cells (D) transfected with vector, ZNF277 plasmid, NC, and siZNF277. Representative images of the invasion assays are shown. The data are presented as mean \pm SD of three independent experiments. $* P<0.05$ as compared with the control group.

Abbreviations: OC, ovarian cancer; NC, negative control.

whether the promotion of $\mathrm{OC}$ cell migration and invasion by ZNF277 was mediated by targeting PTEN. As shown in Figure 6A and B, when SKOV3 and OVCAR3 were transfected with PTEN, the migratory ability was significantly decreased compared with that of the control group, whereas PTEN restoration counteracted the promotion of migrated SKOV3 and OVCAR3 cells by ZNF277. The effect of PTEN on OC cell invasion was also evaluated. Transwell 


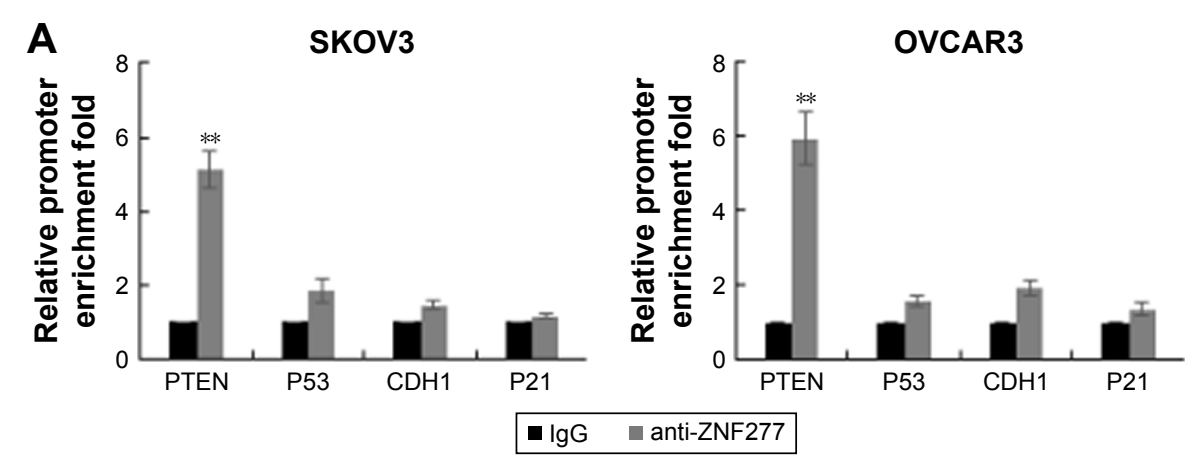

B
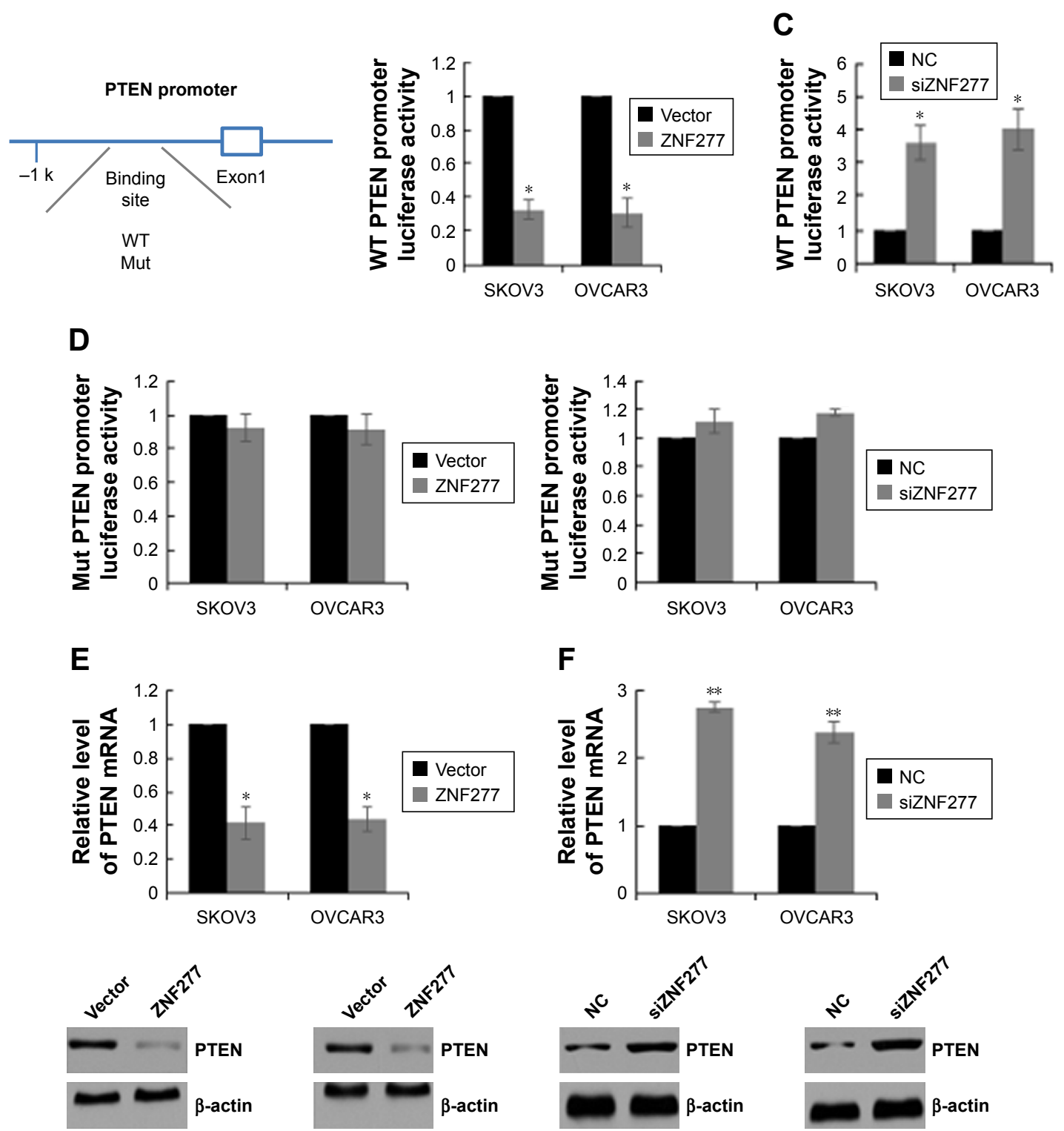

Figure 4 PTEN was a direct downstream target of ZNF277 in OC cells. (A) The binding of ZNF277 to the promoter of PTEN. qChIP was performed in SKOV3 and OVCAR3 cells to identify key target genes for ZNF277, such as P53, PTEN, CDHI, and P2I. Normal IgG was used as a negative control (**P $<0.0 \mathrm{I}$ as compared with the normal $\operatorname{lgG}$ ). (B) Luciferase reporter activities driven by wild-type PTEN promoter were examined in SKOV3 or OVCAR3 cells transfected with Vector or ZNF277. (C) Luciferase reporter activities driven by wild-type PTEN promoter were examined in SKOV3 or OVCAR3 cells transfected with NC or siZNF277. Error bars indicate mean \pm SD of three independent experiments ( $* P<0.05$ vs Vector). (D) Luciferase reporter activities driven by mutant (Mut)-type PTEN promoter were examined in SKOV3 or OVCAR3 cells transfected with vector or ZNF277, NC or siZNF277. (E) Vector or ZNF277 was transfected into SKOV3 and OVCAR3 cells, and then the expression levels of PTEN were monitored using RT-qPCR and Western blot. The experiment was performed in triplicate and subsequently subjected to statistical analysis. (F) NC or siZNF277 was transfected into SKOV3 and OVCAR3 cells, and then the expression levels of PTEN were monitored using RT-qPCR and Western blot. Error bars indicate mean \pm SD of three independent experiments $(* P<0.05$, $* * P<0.0$ I vs $N C)$.

Abbreviations: OC, ovarian cancer; RT-qPCR, quantitative reverse-transcription PCR; qChIP, quantitative chromatin immunoprecipitation; NC, negative control; WT, wild type; Mut, mutant type. 

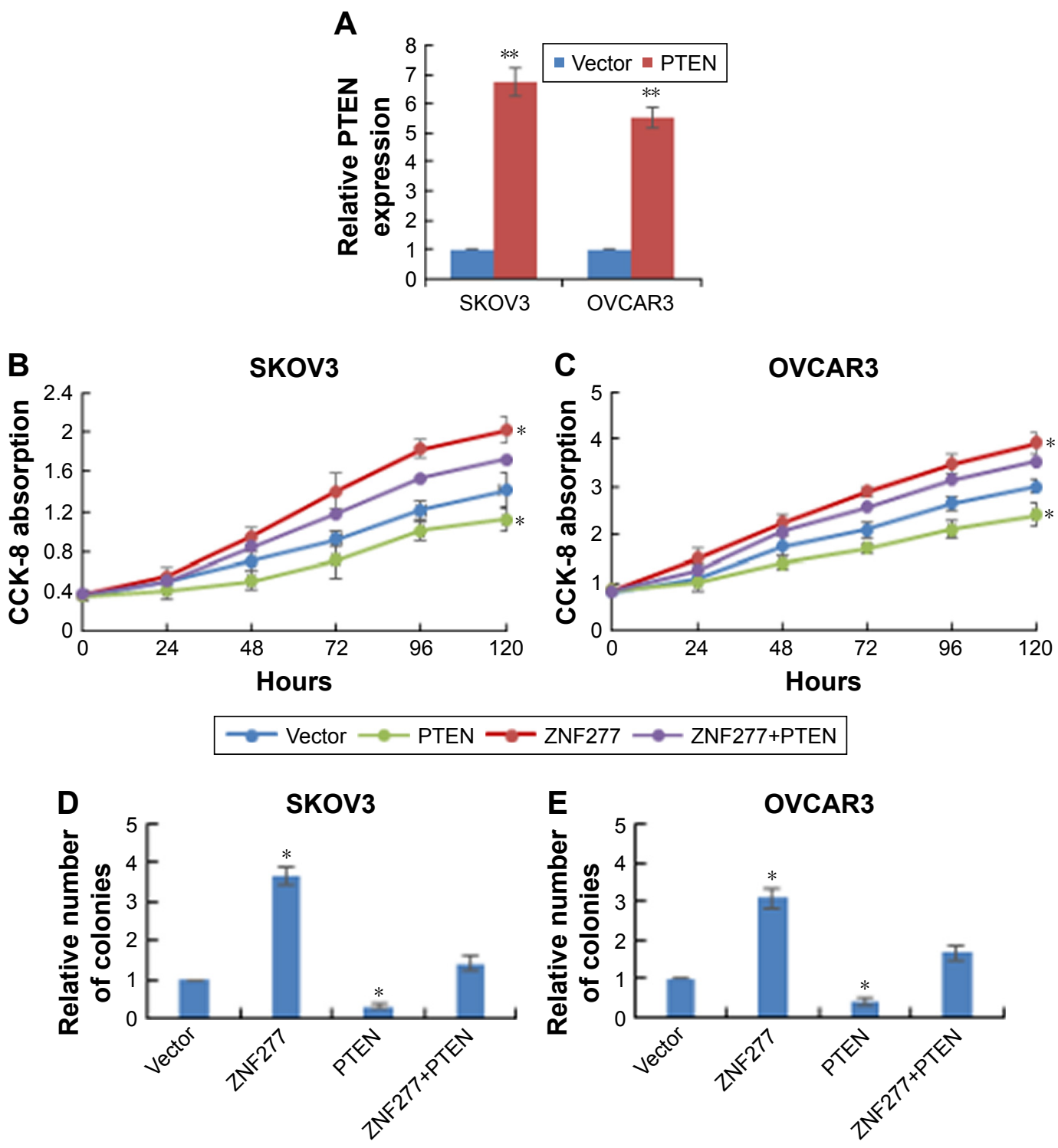

Figure 5 ZNF277 promoted OC cell proliferation by targeting PTEN. (A) The mRNA expression of PTEN was determined by RT-qPCR in SKOV3 and OVCAR3 cells transfected with PTEN overexpression plasmid. Error bars indicate mean \pm SD of three independent experiments (**P $<0.05$ vs vector). (B) Cell viability of SKOV 3 cells and (C) OVCAR3 cells transfected with vector, PTEN, ZNF217, and ZNF2 I7+PTEN was determined by CCK-8 assay. Error bars represent mean \pm SD of three independent experiments ( ${ }^{*} P<0.05$ vs vector). (D) Cell growth of SKOV3 cells and (E) OVCAR3 cells transfected with vector, PTEN, ZNF2I7, and ZNF2I7+PTEN was determined by colony formation assay. Error bars represent mean $\pm S D$ of three independent experiments. ${ }^{*} P<0.05$ vs vector.

Abbreviations: OC, ovarian cancer; RT-qPCR, quantitative reverse-transcription PCR; CCK-8, Cell Counting Kit-8.

assay showed the number of invasive cells was significantly decreased by PTEN overexpression. Analogously, the increased invasion of ZNF277 cells was reduced by PTEN overexpression (Figure 6C and D).

\section{Discussion}

Thus far, not only literature but also gene bank searches have revealed little regarding the function of ZNF277. Although ZNF277 overexpression is reported in chronic lymphocytic leukemia, colon neoplasia, and endocrine tumors, ${ }^{15}$ the role of ZNF277 in OC still remains elusive. Herein, we demonstrate the important role of ZNF277 in promotion of OC. OC tissues and cell lines exhibited increased expression of ZNF277 compared with the normal tissues and cell lines. Furthermore, using TCGA database, survival analysis showed that higher level of ZNF277 was closely associated with poorer overall survival of OC patients. Therefore, ZNF277 might play an oncogenic role in OC. The cells transfected with ZNF277 exhibited significantly increased viability and proliferation, while knockdown of ZNF277 markedly decreased the growth rate. We also found that ZNF277 could regulate the migration and invasion of OC cells.

The $\mathrm{C}_{2} \mathrm{H}_{2}$-type zinc finger motif of ZNF277 is found in numerous transcription factors, ${ }^{18}$ and the capacity of the 

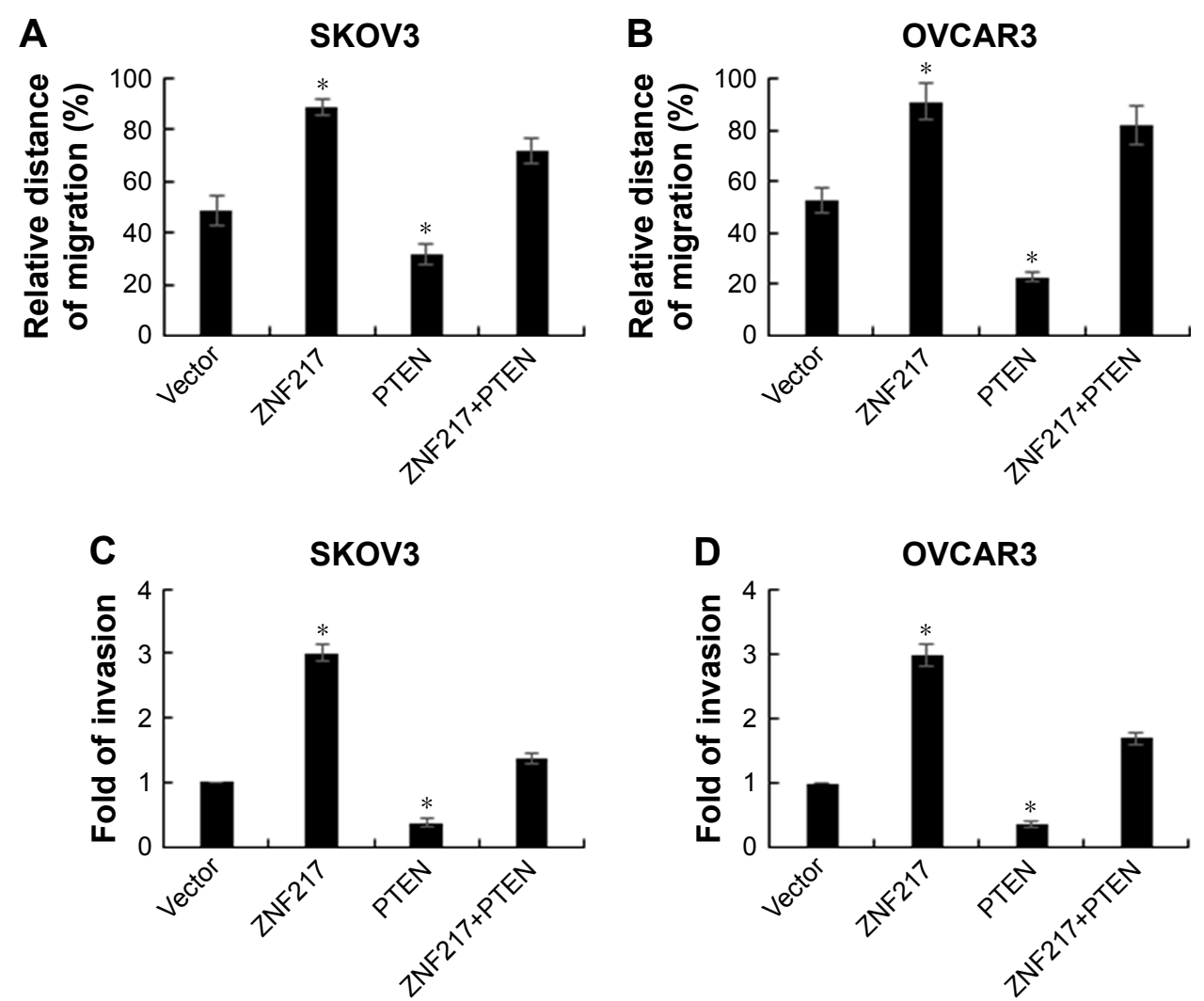

Figure 6 ZNF277 regulated OC cell migration and invasion through PTEN. (A) ZNF277 regulated OC cell (SKOV3) migration through PTEN (*P $<0.05$ vs vector). (B) OVCAR3 cells were transfected with vector, PTEN, ZNF217, and ZNF217+PTEN, respectively, and wound healing assay was performed; error bars represent the mean \pm SD of three independent experiments ( ${ }^{* P}<0.05$ vs vector). (C) ZNF277 regulated OC cell (SKOV3) invasion through PTEN $(* P<0.05$ vs vector). (D) OVCAR3 cells were transfected with vector, PTEN, ZNF217, and ZNF2 I7+PTEN, respectively, and transwell assay was performed accordingly. Error bars represent mean \pm SD of three independent experiments $(* P<0.05$ vs vector).

Abbreviation: OC, ovarian cancer.

zinc finger motif is to bind specific DNA elements. Here, we reported ZNF277 could directly bind to the promoter of PTEN. We identified PTEN as the downstream target gene of ZNF277. The evidence was as follows: overexpression or suppression of ZNF277 could significantly influence the luciferase activity of wild-type promoter of PTEN, while little effect was observed on the mutant promoter of PTEN. Overexpression of ZNF277 resulted in significantly inhibited mRNA and protein level of PTEN, while knockdown of ZNF277 led to obviously increased PTEN expression. Although a panel of putative tumor suppressors were also measured upon ZNF277 overexpression or knockdown, only PTEN expression was shown to be significantly altered. By downregulating the targeted PTEN, ZNF277 may activate the PI3K/Akt signaling pathway, resulting in the OC cell proliferation and invasion. Additionally, the link between ZNF277 and PTEN has rarely been established before. To our knowledge, our study represents the first evidence that ZNF277 suppresses PTEN in OC. PTEN is a wellknown tumor suppressor which plays an important role in the development of various human cancers. ${ }^{6,9}$ It is widely known that loss of PTEN could enhance cancer cell proliferation, migration, and invasion, thereby leading to poor prognosis of patients with OC. ${ }^{19,20}$ Given the significant roles of PTEN, we conclude that ZNF277 is an important molecule in OC. These data also indicate that PTEN is not only the downstream target of ZNF277 but also the functional mediator of ZNF277 in $\mathrm{OC}$, and might provide a potential targeted therapy for the treatment of OC. In the future, further investigations are warranted to explain the clinical utility of ZNF277.

\section{Availability of data and materials}

The datasets used and/or analyzed during the current study are available from the corresponding author on reasonable request.

\section{Disclosure}

The authors report no conflicts of interest in this work.

\section{References}

1. Lynch HT, Casey MJ, Lynch J, White TE, Godwin AK. Genetics and ovarian carcinoma. Semin Oncol. 1998;25(3):265-280. 
2. Siegel R, Naishadham D, Jemal A. Cancer statistics, 2012. CA Cancer J Clin. 2012;62(1):10-29. doi:10.3322/caac.20138

3. Yang L, Zhang B, Xing G, et al. Neoadjuvant chemotherapy versus primary debulking surgery in advanced epithelial ovarian cancer: a metaanalysis of peri-operative outcome. PLoS One. 2017;12(10):e0186725. doi:10.1371/journal.pone. 0186725

4. Kim JY, Cho CH, Song HS. Targeted therapy of ovarian cancer including immune check point inhibitor. Korean J Intern Med. 2017;32(5): 798-804. doi:10.3904/kjim.2017.008

5. McGuire WP. Maintenance therapy for ovarian cancer: of Helsinki and Hippocrates. J Clinl Oncol. 2009;27(28):4633-4634. doi:10.1200/ JCO.2009.23.6653

6. Di Cristofano A, Pandolfi PP. The multiple roles of PTEN in tumor suppression. Cell. 2000;100(4):387-390.

7. Chalhoub N, Baker SJ. PTEN and the PI3-kinase pathway in cancer. Annu Rev Pathol. 2009;4:127-150. doi:10.1146/annurev.pathol. 4.110807.092311

8. Cai J, Xu L, Tang H, et al. The role of the PTEN/PI3K/Akt pathway on prognosis in epithelial ovarian cancer: a meta-analysis. Oncologist. 2014;19(5):528-535. doi:10.1634/theoncologist.2013-0333

9. Carnero A, Blanco-Aparicio C, Renner O, Link W, Leal JF. The PTEN/ PI3K/AKT signalling pathway in cancer, therapeutic implications. Curr Cancer Drug Targets. 2008;8(3):187-198.

10. Suo HB, Zhang KC, Zhao J. MiR-200a promotes cell invasion and migration of ovarian carcinoma by targeting PTEN. Eur Rev Med Pharmacol Sci. 2018;22(13):4080-4089. doi:10.26355/eurrev_201807_15398

11. Liu DT, Yao HR, Li YY, Song YY, Su MY. MicroRNA-19b promotes the migration and invasion of ovarian cancer cells by inhibiting the PTEN/ AKT signaling pathway. Oncol Lett. 2018;16(1):559-565. doi:10.3892/ ol.2018.8695

12. Liu H, Pan Y, Han X, Liu J, Li R. MicroRNA-216a promotes the metastasis and epithelial-mesenchymal transition of ovarian cancer by suppressing the PTEN/AKT pathway. Onco Targets Ther. 2017;10: 2701-2709. doi:10.2147/OTT.S114318
13. Liang H, Guo W, Nagarajan L. Chromosomal mapping and genomic organization of an evolutionarily conserved zinc finger gene ZNF277. Genomics. 2000;66(2):226-228. doi:10.1006/geno.2000.6198

14. Negishi M, Saraya A, Mochizuki S, et al. A novel zinc finger protein Zfp277 mediates transcriptional repression of the Ink4a/arf locus through polycomb repressive complex 1. PLoS One. 2010;5(8):e12373. doi:10.1371/journal.pone.0012373

15. Cheng K, Xie G, Khurana S, et al. Divergent effects of muscarinic receptor subtype gene ablation on murine colon tumorigenesis reveals association of M3R and zinc finger protein 277 expression in colon neoplasia. Mol Cancer. 2014;13:77. doi:10.1186/1476-4598-13-77

16. Livak KJ, Schmittgen TD. Analysis of relative gene expression data using real-time quantitative PCR and the 2(-Delta Delta $\mathrm{C}(\mathrm{T})$ ) method. Methods. 2001;25(4):402-408. doi:10.1006/meth.2001.1262

17. Uhlen M, Bjorling E, Agaton C, et al. A human protein atlas for normal and cancer tissues based on antibody proteomics. Mol Cell Proteomics. 2005;4(12):1920-1932. doi:10.1074/mcp.M500279-MCP200

18. Huang HN, Huang WC, Lin CH, Chiang YC, Huang HY, Kuo KT. Chromosome 20q13.2 ZNF217 locus amplification correlates with decreased E-cadherin expression in ovarian clear cell carcinoma with PI3K-Akt pathway alterations. Hum Pathol. 2014;45(11):2318-2325. doi:10.1016/j.humpath.2014.07.020

19. Westin SN, Ju Z, Broaddus RR, et al. PTEN loss is a context-dependent outcome determinant in obese and non-obese endometrioid endometrial cancer patients. Mol Oncol. 2015;9(8):1694-1703. doi:10.1016/j. molonc.2015.04.014

20. Liu Z, Ren YA, Pangas SA, et al. FOXO1/3 and PTEN depletion in granulosa cells promotes ovarian granulosa cell tumor development Mol Endocrinol. 2015;29(7):1006-1024. doi:10.1210/me.2015-1103 


\section{Supplementary material}

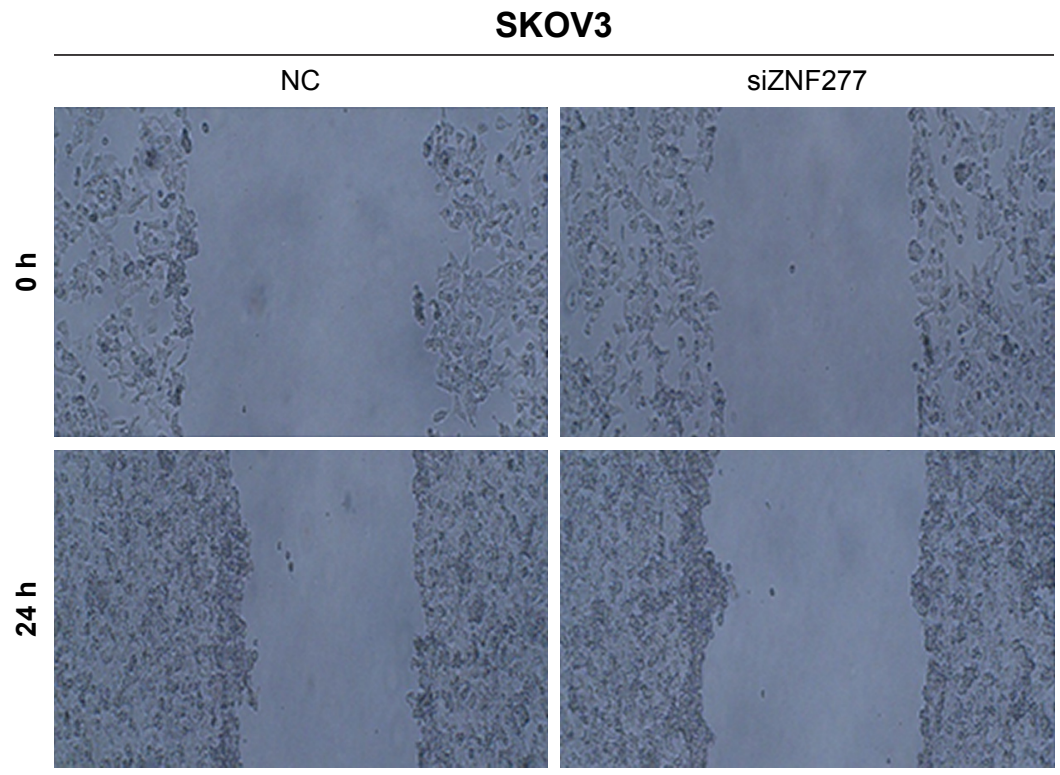

Figure SI ZNF277 promoted migration in OC cells. Wound healing assay was performed in SKOV3 cells following transfection with NC and siZNF277. Pictures of the wound closure rate are shown.

Abbreviations: OC, ovarian cancer; NC, negative control.

\section{Publish your work in this journal}

OncoTargets and Therapy is an international, peer-reviewed, open access journal focusing on the pathological basis of all cancers, potential targets for therapy and treatment protocols employed to improve the management of cancer patients. The journal also focuses on the impact of management programs and new therapeutic agents and protocols on patient perspectives such as quality of life, adherence and satisfaction. The manuscript management system is completely online and includes a very quick and fair peer-review system, which is all easy to use. Visit http://www.dovepress.com/testimonials.php to read real quotes from published authors. 